\title{
A GARCH Approach to VaR Calculation in Financial Market
}

\author{
Nurfadhlina Abdul Halim ${ }^{a^{*}}$, Endang Soeryana ${ }^{b}$, Alit Kartiw \\ ${ }^{a}$ Faculty of Science and Technology, Universiti Sains Islam Malaysia, MALAYSIA \\ ${ }^{b}$ Mathematics Department, Faculty of Mathematics and Natural Sciences, Universitas Padjadjaran, \\ INDONESIA \\ *Corresponding author email: nurfadhlina@usim.edu.my
}

\begin{abstract}
Value at Risk (VaR) has already becomes a standard measurement that must be carried out by financial institution for both internal interest and regulatory. VaR is defined as the value that portfolio will loss with a certain probability value and over a certain time horizon (usually one or ten days). In this paper we examine of $\mathrm{VaR}$ calculation when the volatility is not constant using generalized autoregressive conditional heteroscedastic $(\mathrm{GARCH})$ model. We illustrate the method to real data from Indonesian financial market that is the stock of PT. Indosat Tbk.
\end{abstract}

Keywords: Value at Risk, Risk Management, GARCH model, skewness, kurtosis, Quantile

\section{Introduction}

There are some types of financial market risk, i.e. credit risk, operational risk and market risk. Value at Risk (VaR) is mainly concerned with market risk; however the concept can be use for difference type of risks. $\mathrm{VaR}$ is single estimator of an institution position quantity decline of profit risk category on the market in the share period. This measure might be applied by the institution for estimating the risk and regulatory committee in this case for analyzing the investment opportunity (Jorion, 2004; Alexader, 1999).

$\mathrm{VaR}$ in term of the financial institution is defined as a maximum lost on period of financial position with a certain probability. $\mathrm{VaR}$ is considered as a lost measurement related to an extraordinary event under the standard market condition. For the regulatory committee, VaR is defined as a minimum lost under an extraordinary market condition. These two definitions has a similar based on VaR measurement, however the concept seems different (Tsay, 2005; Dowd, 
2002).

In general, $\mathrm{VaR}$ calculation usually uses econometrics time series models. In this paper, GARCH model is used for the volatility estimating and the VaR calculation worked base on quantile. In seeking the performance of the models, we'll discuss them through the skewness and kurtosis coefficient values.

\section{Mathematical Models}

\subsection{Value at Risk}

Assume that at the time index $t$ we are concerned in the risk of a financial position for the next $l$ periods. Let $\Delta V(l)$ be the change in value of the asset in financial position from time $t$ to $t+l$. This quantity is measured in rupiah curency and is a random variable at the time index $t$. Represent the cumulative distribution function (CDF) of $\Delta V(l)$ with $F_{l}(x)$. Difined the VaR of a long position over the time horizon $l$ with probability $p$ as

$$
p=\operatorname{Pr}[\Delta V(l) \leq \mathrm{VaR}]=F_{l}(\mathrm{VaR})
$$

Because the holder of a long financial position suffers a loss when $\Delta V(l)<0$, the $\mathrm{VaR}$ defined in (1) naturally assumes a negative value when $p$ is small. The negative sign signifies a loss. From the definition, the probability that the holder would run into a loss greater than or equal to $\mathrm{VaR}$ over the time horizon $l$ is $p$. Alternatively, VaR can be interpreted as follow. With probability $(1-p)$, the potential loss encountered by the holder of the financial position over the time horizon $l$ is less than or equal to VaR.

The holder of a short position suffers a loss when the value of the asset increases $\Delta V(l)>0$. The $\mathrm{VaR}$ is then defined as

$$
p=\operatorname{Pr}[\Delta V(l) \geq \mathrm{VaR}]=1-\operatorname{Pr}[\Delta V(l) \leq \mathrm{VaR}]=1-F_{l}(\mathrm{VaR})
$$

For a small $p$, the VaR of a short position naturally assumes a positive value. The positive sign signifies a loss. The previous definitions show that VaR is concerned with tail behavior of the CDF $F_{l}(x)$. For a long position, the left tail of $F_{l}(x)$ is important. Nevertheless a short position focuses on the right tail of $F_{l}(x)$. The definition of $\mathrm{VaR}$ in (1) continues to apply to a short position if one uses the distribution of $-\Delta V(l)$. Therefore, it suffices to discuss method of VaR calculation using a long position (Tsay, 2005; Khindanova).

For any univariate $\mathrm{CDF} F_{l}(x)$ and probability $p$, such that $0<p<1$, the quantity

$$
x_{p}=\inf \left\{x \mid F_{l}(x) \geq p\right\}
$$

Is called the $p$ th quantile of $F_{l}(x)$, where inf denotes the smallest real number satisfying $F_{l}(x) \geq p$. If the $\mathrm{CDF} F_{l}(x)$ of (1) is known, then $\mathrm{VaR}=x_{p}$. However usually the CDF is 
unknown in practice, then the studies of VaR are essentially concerned with estimation of the CDF and or its quantile, especially the tail behavior of the CDF.

In practical applications, calculation of VaR involves several factors:

1. The probability of interest $p$, such as $p=0.01$ or $p=0.05$.

2. The time horizon $l$. It might be set by a regulatory committee, such as 1 day or 10 days.

3. The frequency of the data, which might not be the same as the time horizon $l$. Daily observations are often used.

4. The $\mathrm{CDF} F_{l}(x)$ or its quantiles.

5. The amount of the financial position or the mark-to-market value of the portfolio.

Among these factors, the $\mathrm{CDF} F_{l}(x)$ is the focus of econometric modeling.

\subsection{The GARCH Approach}

For a log return series, the time series models can be used to model the mean equation, and conditional heteroscedastic models are used to handle the volatility. In this paper, we will use GARCH models to the approach as an econometric approach to VaR calculation (Tsay, 2005; Engle \& Manganelli, 2002).

Consider the log return $r_{t}$ of an asset. A general time series model for $r_{t}$ can be written as

$$
\begin{gathered}
r_{t}=\phi_{0}+\sum_{i=1}^{p} \phi_{i} r_{t-i}+a_{t}-\sum_{j=1}^{q} \theta_{j} a_{t-j} \\
a_{t}=\sigma_{t} \varepsilon_{t}, \\
\sigma_{t}^{2}=\alpha_{0}+\sum_{i=1}^{u} \alpha_{i} a_{t-i}^{2}+\sum_{j=1}^{v} \beta_{j} \sigma_{t-j}^{2}
\end{gathered}
$$

If one further assumes that $\varepsilon_{t}$ is Gaussian, then the conditional distribution of $r_{t+1}$ given the information available at time $t$ is $N\left[\hat{r}_{t}(1), \hat{\sigma}^{2}(1)\right]$. Quantiles of this conditional distribution can easily be obtained for $\mathrm{VaR}$ calculation. For example, the $5 \%$ quantile is $\hat{r}_{t}(1)-1.65 \hat{\sigma}_{t}(1)$. If one assumes that $\varepsilon_{t}$ is a standardized Student- $t$ distribution with $v$ degrees of freedom, then the quantile is $\hat{r}_{t}(1)-t_{v}^{*}(p) \hat{\sigma}_{t}(1)$, where $t_{v}^{*}(p)$ is the $p$ th quantile of standardized student- $t$ distribution with $v$ degrees of freedom.

The relationship between quantiles of a Student- $t$ distribution with $v$ degrees of freedom, denoted by $t_{v}$, and those of its standardized distribution, denoted by $t_{t}^{*}$, is

$$
p=\operatorname{Pr}\left(t_{v} \leq q\right)=\operatorname{Pr}\left(\frac{t_{v}}{\sqrt{v /(v-2)}} \leq \frac{q}{\sqrt{v /(v-2)}}\right)=\operatorname{Pr}\left(t_{v}^{*} \leq \frac{q}{\sqrt{v /(v-2)}}\right)
$$

where $v>2$. That is, if $q$ is $p$ th quantile of a Student- $t$ distribution with $v$ degrees of freedom, 
then $q / \sqrt{v /(v-2)}$ is the $p$ th quantile of a standardized Student- $t$ distribution with $v$ degrees of freedom. Therefore, if $\varepsilon_{t}$ of the GARCH model (5) is a standardized Student- $t$ distribution with $v$ degrees of freedom and the probability is $p$, then the quantile used to calculate the 1-period horizon $\mathrm{VaR}$ at time index $t$ is

$$
\hat{r}_{t}(1)+\frac{t_{v}(p) \hat{\sigma}_{t}(1)}{\sqrt{v /(v-2)}}
$$

Where, $t_{v}(p)$ is the $p$ th quantile of a Student- $t$ distribution with $v$ degrees of freedom and assumes a negative value for a small $p$.

\subsubsection{The Model Parameter Estimation}

Let $\mathrm{L}(r ; \theta)$ is denote the conditional likelihood function concerned with $r_{t}$. The likelihood function of $r_{1}, \ldots, r_{T}$ conditional on $r_{0}$ is

$$
\mathrm{L}(r ; \theta)=\prod_{i=1}^{T} \mathrm{~L}_{i}(r ; \theta)
$$

Therefore the estimator is a solution for maximize the problem

$$
\max _{\theta} \log \mathrm{L}(r ; \theta)
$$

This estimator denoted by $\hat{\theta}_{T}$ and is called pseudo maximum likelihood estimator (PMLE), and the likelihood function is constructed by the assume that a normally distribution. When the distribution assumption is not true, this estimator is a normal asymptotically with the covariance matrix is

$$
\begin{gathered}
V_{a s}\left[\sqrt{T}\left(\hat{\theta}_{T}-\theta\right)\right]=\mathbf{J}^{-\mathbf{1}} \mathbf{I} \mathbf{J}^{-\mathbf{1}} \\
\mathbf{J}=E_{0}\left[-\frac{\partial^{2} \log \mathrm{L}_{i}(r ; \theta)}{\partial \theta \cdot \partial \theta^{\prime}}\right] \\
\mathbf{I}=E_{0}\left[\frac{\partial \log \mathrm{L}_{i}(r ; \theta)}{\partial \theta} \frac{\partial \log \mathrm{L}_{i}(r ; \theta)}{\partial \theta^{\prime}}\right]
\end{gathered}
$$

where $E_{0}$ showed that the expectation taken to the true distribution (Gourieoux, 2002).

Two matrix $\mathbf{I}$ and $\mathbf{J}$ is usually different. But $\mathbf{I}$ and $\mathbf{J}$ is equal if its distribution is true and appropriate with the likelihood function, that is in this case a conditional normal distribution. When the matrix $\quad \mathbf{I}=\mathbf{J}$, the asymptotic patern becomes simple, that is

$$
V_{a s}\left[\sqrt{T}\left(\hat{\theta}_{T}-\theta\right)\right]=\mathbf{J}^{-\mathbf{1}}=\mathbf{I}^{-\mathbf{1}}
$$

On the GARCH model, notified that the MLE of ARMA model usually solved by using the backward forecast algorithm or Kalman filter. The similar case when the ARCH specification is replaced by $\operatorname{GARCH}$. Let given a $\operatorname{GARCH}(p, q)$ model conditionally Gaussian

$$
r_{t} \mid r_{t-1} \sim N\left[0, \sigma_{t}^{2}\right]
$$


where $\sigma_{t}^{2}$ given in (5).

The conditional variance patern in the parameter terms and the variable observed is

$$
\sigma_{t}^{2}=\frac{1}{1-\sum_{j=1}^{q} \beta_{j} L^{j}}\left(c+\sum_{i=1}^{p} \alpha_{i} r_{t-i}^{2}\right)
$$

where $L$ represent the operator-lag. Hence, $\sigma_{t}^{2}(\theta)$ depend on the all previous values of $r_{t}$ process.

Since the time period of observation is limited, that is $t=1, \ldots, T$, here need to replace $\sigma_{t}^{2}(\theta)$ with truncated approximattion, where $r_{t}^{2}$ is values that concerned with negative sign that defined equal to zero. It is equivalent with the recursive equation

$$
\hat{\sigma}_{t}^{2}=c+\sum_{i=1}^{p} \alpha_{i} \bar{r}_{t-i}^{2}+\sum_{j=1}^{q} \beta_{j} \hat{\sigma}_{t-j}^{2}
$$

with

$$
\bar{r}_{t}=0 \text {, if } t \leq 0 ; \bar{r}_{t}=r_{t} \text {, if } t \geq 1 \text {; and } \hat{\sigma}_{t}^{2}=0 \text {, if } t \leq 0 \text {. }
$$

The initialy log-likelihood function replaced with truncated version:

$$
\log \tilde{\mathrm{L}}=-\frac{T}{2} \log 2 \pi-\frac{1}{2} \sum_{t=1}^{T} \log \tilde{\sigma}_{t}^{2}(\theta)-\frac{1}{2} \sum_{t=1}^{T} \frac{r_{t}^{2}}{\tilde{\sigma}_{t}^{2}(\theta)}
$$

Continuously, the optimization is worked with numerical procedure and for the value of $\theta_{1}$ from $\theta$ that given, the conditional variance respectively calculated by using

$$
\tilde{\sigma}_{t}^{2}\left(\theta_{1}\right)=c_{1}+\sum_{i=1}^{p} \alpha_{i 1} \tilde{r}_{t-1}^{2}+\sum_{j=1}^{q} \beta_{j 1} \tilde{\sigma}_{t-j}^{2}\left(\theta_{1}\right)
$$

\subsubsection{Diagnistic Check for ARCH Effect}

Rembering that the ARCH model is $a_{t}=\sigma_{t} v_{t}, v_{t} \sim \operatorname{iid} N(0,1)$ or standardized Student- $t$ distribution. By using the "Standardize-Shock" $\hat{v}_{t}=\hat{a}_{t} / \hat{\sigma}_{t}$ where $\hat{\sigma}_{t}$ is calculated by ARCH model that estimated. When the ARCH model used is good adequate $\hat{v}_{t}$ is being equivalent with $v_{t}$ . It can be done by several ways: (1) Plot of the ACF and PACF for $\hat{v}_{t}$; (2) Determine Potmanteu statistic for $\hat{v}_{t}$, if the ARC/GARCH model is appropriate, then Potmanteu statistic is not rejected; and observation can be done one is using the kurtosis toward $\left\{\hat{v}_{t}\right\}$ for seeing appropriation of the chosen assumption (Shi, 2004).

\subsection{Kurtosis of GARCH Models}


To assess the variability of an estimated volatility, one must consider the kurtosis of a volatility model. In this section, we will derive the excess kurtosis of a GARCH(1,1) model. The same idea applies to other GARCH models. The model considered is

$$
a_{t}=\sigma_{t} \varepsilon_{t}, \sigma_{t}^{2}=\alpha_{0}+\alpha_{1} a_{t-1}^{2}+\beta_{1} \sigma_{t-1}^{2}
$$

where $\alpha_{0}>0, \alpha_{1} \geq 0, \beta_{1} \geq 0, \alpha_{0}>0$, and $\left\{\varepsilon_{t}\right\}$ is an iid sequence satisfying

$$
E\left(\varepsilon_{t}\right)=0, \operatorname{Var}\left(\varepsilon_{t}\right)=1, E\left(\varepsilon_{t}^{4}\right)=K_{\varepsilon}+3
$$

Where $K_{\varepsilon}$ is the excess kurtosis of the innovation $\varepsilon_{t}$. Based on the assumption, obtained the following:

- $\operatorname{Var}\left(a_{t}\right)=E\left(\sigma_{t}^{2}\right)=\frac{\alpha_{0}}{1-\left(\alpha_{1}+\beta_{1}\right)}$

- $E\left(a_{t}^{4}\right)=\left(K_{\varepsilon}+3\right) E\left(a_{t}^{4}\right)$ provided that $E\left(a_{t}^{4}\right)$ exists.

Taking the square of the volatility model (5) for $i=1$, obtained

$$
\sigma_{t}^{4}=\alpha_{0}^{2}+\alpha_{1}^{2} a_{t-1}^{4}+\beta_{1}^{2} \sigma_{t-1}^{4}+2 \alpha_{0} \alpha_{1} a_{t-1}^{2}+2 \alpha_{0} \beta_{1} \sigma_{t-1}^{2}+2 \alpha_{1} \beta_{1} \sigma_{t-1}^{2} a_{t-1}^{2}
$$

Taking expectation of the equation (21) and using the two properties mentioned earlier, obtained

$$
E\left(\sigma_{t}^{4}\right)=\frac{\alpha_{0}^{2}\left(1+\alpha_{1}+\beta_{1}\right)}{\left[1-\left(\alpha_{1}+\beta_{1}\right)\right]\left[1-\alpha_{1}^{2}\left(K_{\varepsilon}+2\right)-\left(\alpha_{1}+\beta_{1}\right)^{2}\right]}
$$

Provided that $0 \leq \alpha_{1}+\beta_{1}<1$ and $1-\alpha_{1}^{2}\left(K_{\varepsilon}+2\right)-\left(\alpha_{1}+\beta_{1}\right)^{2}>0$. Excess kurtosis of $a_{t}$, if exists, is

$$
K_{a}=\frac{E\left(a_{t}^{4}\right)}{\left[E\left(a_{t}^{2}\right)\right]^{2}}-3=\frac{\left(K_{\varepsilon}+3\right)\left[1-\left(\alpha_{1}+\beta_{1}\right)^{2}\right]}{1-2 \alpha_{1}^{2}-\left(\alpha_{1}+\beta_{1}\right)^{2}-K_{\varepsilon} \alpha_{1}^{2}}-3
$$

This excess kurtosis can be written in an informative expression. First, consider the case that $\varepsilon_{t}$ is normally distributed. In this case, $K_{\varepsilon}=0$, and some algebra shows that

$$
K_{a}^{(g)}=\frac{6 \alpha_{1}^{2}}{1-2 \alpha_{1}^{2}-\left(\alpha_{1}+\beta_{1}\right)^{2}},
$$

Where, the superscript $(g)$ is used to denote Gaussian distribution. This result has two important implications: (a) the kurtosis $a_{t}$ exists if $1-2 \alpha_{1}^{2}-\left(\alpha_{1}+\beta_{1}\right)^{2}>0$, and (b) if $\alpha_{0}=0$, then $K_{a}^{(g)}=0$, denotation that the corresponding $\operatorname{GARCH}(1,1)$ model does not have heavy tails.

Second, consider the case that $\varepsilon_{t}$ is not Gaussian. Using the previous result, obtained

$$
K_{a}=\frac{K_{\varepsilon}-K_{\varepsilon}\left(\alpha_{1}+\beta_{1}\right)+6 \alpha_{1}^{2}+3 K_{\varepsilon} \alpha_{1}^{2}}{1-2 \alpha_{1}^{2}-\left(\alpha_{1}+\beta_{1}\right)^{2}-K_{\varepsilon} \alpha_{1}^{2}}
$$




$$
\begin{aligned}
& =\frac{K_{\varepsilon}\left[1-2 \alpha_{1}^{2}-\left(\alpha_{1}+\beta_{1}\right)^{2}\right]+6 \alpha_{1}^{2}+5 K_{\varepsilon} \alpha_{1}^{2}}{1-2 \alpha_{1}^{2}-\left(\alpha_{1}+\beta_{1}\right)^{2}-K_{\varepsilon} \alpha_{1}^{2}} \\
& =\frac{K_{\varepsilon}+K_{a}^{(g)}+\frac{5}{6} K_{\varepsilon} K_{a}^{(g)}}{1-\frac{1}{6} K_{\varepsilon} K_{a}^{(g)}}
\end{aligned}
$$

It holds for all GARCH models provided that the kurtosis exists. For instance, if $\beta_{1}=0$, then the model reduces to an $\mathrm{ARCH}(1)$ model. In this case, it is easy to verify that $K_{a}^{(g)}=6 \alpha_{1}^{2} /\left(1-3 \alpha_{1}^{2}\right)$ provided that $1>3 \alpha_{1}^{2}$ and the excess kurtosis of $a_{t}$ is

$$
\begin{aligned}
K_{a}= & \frac{\left(K_{\varepsilon}+3\right)\left(1-\alpha_{1}^{2}\right)}{1-\left(K_{\varepsilon}+3\right) \alpha_{1}^{2}}-3=\frac{K_{\varepsilon}+2 K_{\varepsilon} \alpha_{1}^{2}+6 \alpha_{1}^{2}}{1-3 \alpha_{1}^{3}-K_{\varepsilon} \alpha_{1}^{2}} \\
= & \frac{K_{\varepsilon}\left(1-3 \alpha_{1}^{2}\right)+6 \alpha_{1}^{2}+5 K_{\varepsilon} \alpha_{1}^{2}}{1-3 \alpha_{1}^{2}-K_{\varepsilon} \alpha_{1}^{2}} \\
& =\frac{K_{\varepsilon}+K_{a}^{(g)}+\frac{5}{6} K_{\varepsilon} K_{a}^{(g)}}{1-\frac{1}{6} K_{\varepsilon} K_{a}^{(g)}}
\end{aligned}
$$

The previous result shows that for a GARCH(1,1) model the coefficient $\alpha_{1}$ plays a critical role in determining the tail behavior of $a_{t}$. If $\alpha_{1}=0$, then $K_{a}^{(g)}=0$ and $K_{a}=K_{\varepsilon}$. In this case, the tail behavior of $a_{t}$ is similar to that of the standardized noise $\varepsilon_{t}$. Nevertheless if $\alpha_{1}>0$, then $K_{a}^{(g)}>0$ and the $a_{t}$ process has heavy tails (Tsay, 2005; Shi, 2004).

For a standadized Student- $t$ distribution with $v$ degrees of freedom, obtained $E\left(\varepsilon_{t}^{4}\right)=6 /(v-4)+3$ if $v>4$. Therefore, the excess kurtosis of $\varepsilon_{t}$ is $K_{\varepsilon}=6 /(v-3)$ for $v>4$. This is part of the reason that used $t_{5}$ when the degrees of freedom of a student- $t$ distribution are prespecified. The excess kurtosis of $a_{t}$ becomes $K_{a}=\left[6+(v+1) K_{a}^{(g)}\right] /\left[v-4-K_{a}^{(g)}\right]$ provided that $1-2 \alpha_{1}^{2}(v-1) /(v-4)-\left(\alpha_{1}+\beta_{1}\right)^{2}>0$.

\section{Cases Study}

The aim of this research is to determine the Value at Risk (VaR) of the real data. As a case study, we use PT. Indosat, Tbk. stock data. In this research, we employ GARCH model to estimate this VaR value. The result of this approach can be used by investors to save their stocks. We implement MATLAB 7.1 and EView 3 for analyzing the data. 
The data which will be analyzed are log return data (Continuously Compounded Return) of closing price. We observe the data from July, $4^{\text {th }}, 2004$ till March, $3^{\text {rd }} 2006$, or contain 396 observations. Time series plot of the data can be seen at Figure 3.1 as follow:

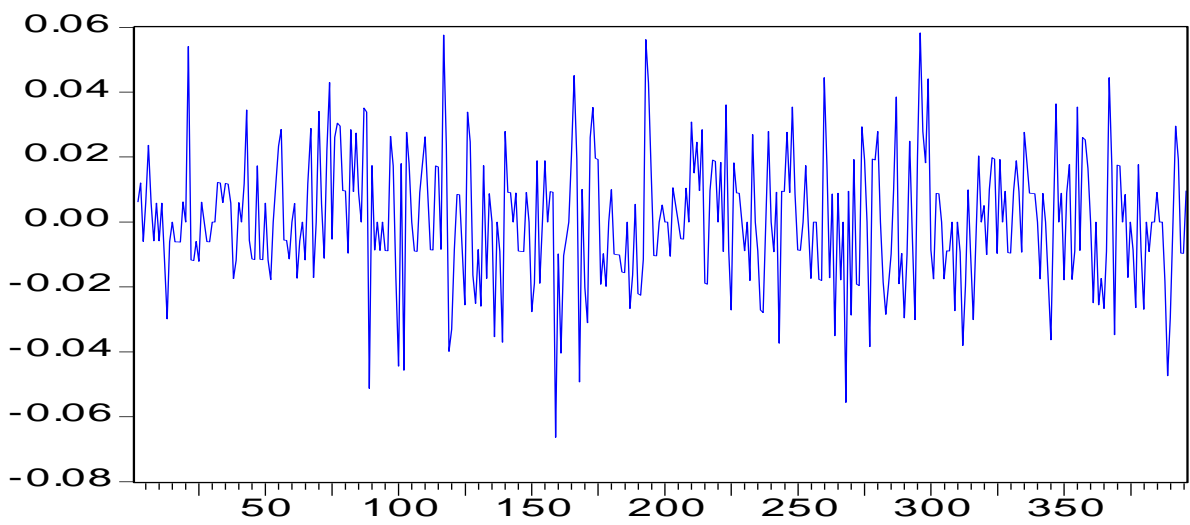

Figure 1: Plot of Log Return the transaction from July $4^{\text {th }}, 2004$ to March $3^{\text {rd }}, 2006$

The summary of descriptive statistics for the data is as follows: the minimum is -0.066375 , the maximum is 0.058269 , mean is 0.000602 , variance is 0.000385 , and standard deviation is 0.019634 .

\subsection{Normality Test}

We employ MINITAB 13 to do normality test with the hypothesis test as follow

$\mathrm{H}_{0}$ : The log return data are normally distributed

$\mathrm{H}_{1}$ : The log return data are not normally distributed.

The hypothesis null is rejected if p-value of the statistic test less than 0.05 (significance level). In this paper, we use Ryan-Joiner and Shapiro-Wilk Test as statistic tests. The results show that pvalue of both tests are the same, i.e. 0.081 . It means we fail to reject $\mathrm{H}_{0}$ and conclude that log return data are normally distributed.

\subsection{GARCH Modeling}

In this section we will estimate whether the $\log$ return data have volatility pattern following GARCH model. Identification step by using time series plot (see Figure 3.1) shows that data satisfy stationery condition in mean. Both of the ACF and PACF indicate only significant at the first lag or cut off after lag 1. Based on this result, we can propose that the appropriate ARIMA models are $\mathrm{AR}(1)$ or MA(1). The results of parameter estimation and diagnostic check steps show that MA(1) model is the best appropriate model. The output of MA(1) model is illustrated at Table 3.1 as follow: 
Table 1: Moving Average orde 1 modeling parameter estimation

\begin{tabular}{|c|c|c|c|c|}
\hline $\begin{array}{l}\text { Dependent Variable: } \\
\text { Method: Least Squal } \\
\text { Date: 04/20/06 Tim } \\
\text { Sample(adjusted): } 2 \\
\text { Included observation } \\
\text { Convergence achiev } \\
\text { Backcast: } 1\end{array}$ & $\begin{array}{l}\text { OGRET } \\
12: 54 \\
6 \\
395 \text { after adj } \\
\text { after } 5 \text { iterat }\end{array}$ & $\begin{array}{l}\text { sting endpo } \\
\text { ns }\end{array}$ & & \\
\hline Variable & Coefficient & Std. Error & $\mathrm{t}$-Statistic & Prob. \\
\hline $\operatorname{MA}(1)$ & 0.147232 & 0.049853 & 2.953349 & 0.0033 \\
\hline R-squared & 0.017677 & Mean depe & ent var & 0.000602 \\
\hline Adjusted R-squared & 0.017677 & S.D. depen & nt var & 0.019634 \\
\hline S.E. of regression & 0.019460 & Akaike inf & riterion & -5.038365 \\
\hline Sum squared resid & 0.149207 & Schwarz cr & erion & -5.028292 \\
\hline Log likelihood & 996.0770 & Durbin-Wa & on stat & 2.017408 \\
\hline Inverted MA Roots & -.15 & & & \\
\hline
\end{tabular}

Hence, MA(1) model is an appropriate for modeling the mean of log return data of PT. Indosat, Tbk stock. This model can be presented as $\hat{F}\left(r_{t}, t-1\right)=0.147232 a_{t-1}$.

Then, we continue to analyze whether the square of residuals model have heteroscedasticity. In this case, the residuals component are calculated as follows; $\hat{a}_{t}=r_{t}-\hat{F}_{t-1}$, with $r_{t}=\log$ return of actually price at the $t$ time and $\hat{F}_{t-1}=$ forecast value at the $t-1$ time. We apply ARCH-LM test to evaluate it and the results are illustrated at Table 3.2 as follow:

Table 2: ARCH LM test MA(1)

\begin{tabular}{llll} 
ARCH Test: & & \\
\hline \hline F-statistic & 2.398786 & Probability & 0.036810 \\
Obs*R-squared & $=\underline{11.81238}=\underline{\text { Probability }}=\underline{\underline{ }}=\underline{0.037451}$ \\
\hline
\end{tabular}

The results show that residuals model contain ARCH effect or follow heteroscedasticity pattern. The correlogram of residual indicates that lag 1 and 2 are significant. Hence, we propose that $\mathrm{ARCH}(2)$ and $\operatorname{GARCH}(2,2)$ are appropriate for this data.

Parameter estimation step show that $\mathrm{ARCH}(2)$ model is not appropriate for modeling heteroscedasticity pattern at the residuals. It's caused one of parameters model is not statistically significant. Then, we continue to estimate $\operatorname{GARCH}(2,2)$ model and the result can be seen at Table 3.3 as follow: 
Table 3: MA(1)-GARCH(2,2) models parameter estimation

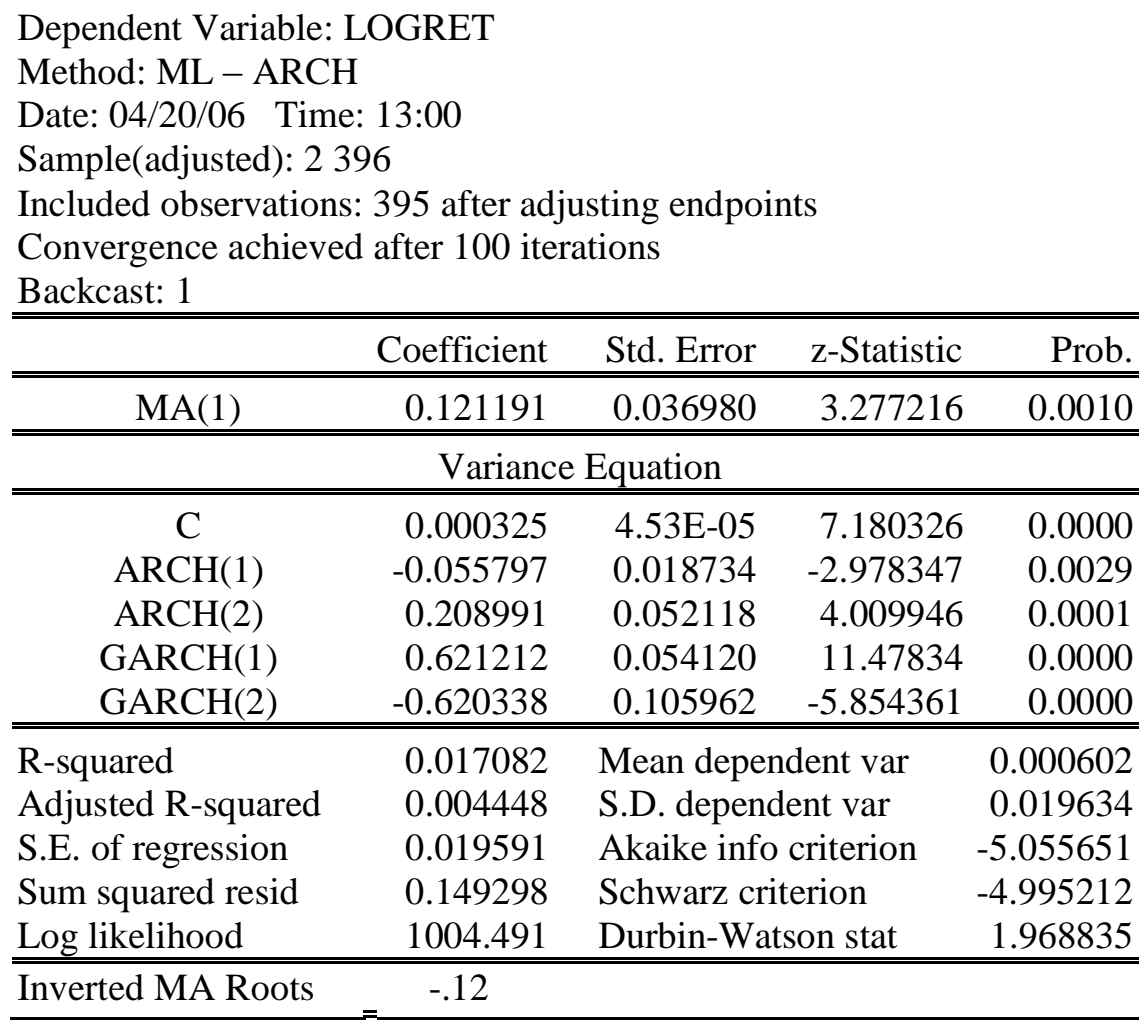

We can observe that all parameters model are significant. Hence, we can conclude that $\operatorname{GARCH}(2,2)$ is the appropriate model for modeling heteroscedasticity pattern at the residuals. This model can be written mathematically as follows $r_{t}=\theta_{1} a_{t-1}$ and $\sigma_{t}^{2}=\omega+\alpha_{1} r_{t-1}^{2}+\alpha_{2} r_{t-2}^{2}+\beta_{1} \sigma_{t-1}^{2}+\beta_{2} \sigma_{t-2}^{2}$. Based on the results for mean and volatility models, we get MA(1)-GARCH(2,2) as follows:

$$
\begin{aligned}
& r_{t}=0,121191 a_{t-1} \\
& \sigma_{t}^{2}=0,000325-0,055797 r_{t-1}^{2}+0,208991 r_{t-2}^{2}+0,621212 \sigma_{t-1}^{2}-0,620338 \sigma_{t-2}^{2}
\end{aligned}
$$

Diagnostic Checking to understand what the model is good enough for the data modeling, we used the ARCH LM test and the result can be seen at Table 3.4 as follow:

Table 4: ARCH LM test

ARCH Test:

\begin{tabular}{llll}
\hline \hline F-statistic & 0.933450 & Probability & 0.502208 \\
Obs*R-squared & 9.375056 & Probability & 0.496917 \\
\hline \hline
\end{tabular}


The result at diagnostic check step is illustrated at Table 3.4. It shows that MA(1)-GARCH(2,2) model satisfies the adequacy model. Additionally, we also check the normality of the residuals model by using Jarque-Berra test, and the result obtained a probability value is 0.358359 geater than of 5\% significance level. It is shows that residuals satisfy normal distribution assumption. Besides that also obtained the coefficient skewness is 0.084146 and the coefficient kurtosis is 3.310456 . Hence, we can conclude that $\mathrm{MA}(1)-\operatorname{GARCH}(2,2)$ is the best model for volatility estimating log return data. The estimated of this model can be seen at Figure 4.8 as follow:

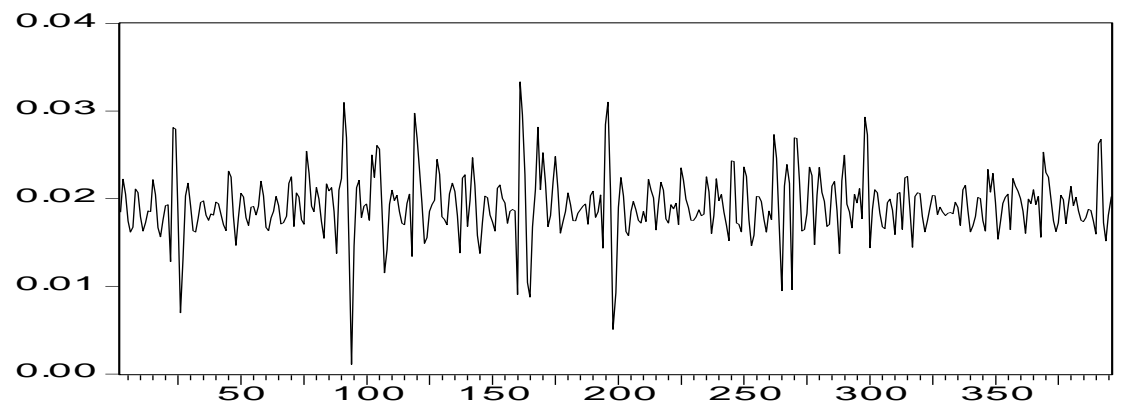

Figure 2: Plot of MA(1)-GARCH(2,2) model volatility

We only need variance model to estimate the volatility. The variance forecast at $397^{\text {th }}$ period by using $\operatorname{GARCH}(2,2)$ model is 0.00041 . It means the daily volatility is 0.0202 .

\subsection{Value at Risk Calculation}

In this paper, the method for calculating $\mathrm{VaR}$ is a variance-covariance approach model. This model has an assumption that return data are normally distributed. From the results at the previous section, we can construct a summary of $\mathrm{VaR}$ values at some level confidence interval, i.e.

Table 5: VaR value at several of the volatility estimation method and cl

\begin{tabular}{|c||c|c|c|c|c|c|}
\hline \multirow{2}{*}{} & \multicolumn{3}{c|}{ Constant Volatility } & \multicolumn{3}{c|}{$M A(1)-G A R C H(2,2)$} \\
Volatilitas & \multicolumn{3}{c|}{0,019634} & \multicolumn{3}{c|}{0,0202} \\
\hline $\mathrm{Cl}$ & $90 \%$ & $95 \%$ & $99 \%$ & $90 \%$ & $95 \%$ & $99 \%$ \\
\hline $\mathrm{VaR}$ & 0.0252 & 0.0323 & 0.0457 & 0.0259 & 0.0332 & 0.047 \\
\hline
\end{tabular}

This result explained if the investor allocate Rp. 100,000,000,00 and invest to PT. Indonsat Tbk. stock by $95 \%$ confidence level, then he will be lost Rp. 3.230.000,- (i.e. $0.0323 \mathrm{x} \mathrm{Rp}$. $100,000,000$,-) based on the Constant Volatility, or Rp. 3,332,000.- (i.e. 0.0332 x Rp. 100,000,000.) based on the GARCH model. Hence, the VaR based on the GARCH model is greater than Constant Volatility approach. 


\section{Conclusion}

Based on the results at the previous section, we can conclude that the log return data of daily closing price of PT. Indosat, Tbk. stock follows the normal distribution. We further obtain that the MA(1)-GARCH(2,2) is the appropriate model for estimating the volatility of this log return data. The model yields the daily volatility forecast around 0.0202. In general, the VaR based on the GARCH model is greater than the Constant Volatility approach.

\section{References}

Alexader, C. (Editor). (1999). Risk Management and Analysis. Volume 1 : Measuring and Modelling Financial Risk. New York : John Wiley \& Sons Inc.

Dowd, K. (2002). An Introduction to Market Risk Measurement. United State American : John Wiley \& Sons Inc.

Engle, R.F. \& Manganelli, S. (2002). CAViaR : Conditional Autoregressive Value at Risk by regression Quantiles.

Gourieroux, C. (1997). ARCH Models and Financial Applications. New York : Springer

Jorion, P. (2004). Bank Trading Risk and Systemic Risk. Third draft : December 2004.

Khindanova, I.N. \& Rachev, S.T. (none). Value at Risk : Recent Advances. University of California, Santa Barbara and University of Karlsruhe, Germany.

Shi-Jie Deng. (2004). Heavy-tailed GARCH Models : Pricing and Risk Management Applications in Power Market. IMA Control \& Pricing in Communication \& Power Networks, 7-13 Mar. 2004.

Tsay, R.S. (2005). Analysis of Financial Time Series. Second Edition. Hoboken, New Jersey : John Wiley \& Sons, Inc. 